\title{
FLUID MECHANICS OF MOLTEN METAL DROPLETS IN ADDITIVE MANUFACTURING
}

\author{
V. TESAŘ \& J. ŠONSKÝ \\ Institute of Thermomechanics Czech Academy of Sciences v.v. i., Prague, Czech Republic.
}

\begin{abstract}
Among basic problems associated with additive manufacturing of metal objects by accretion of moltenmetal droplets is a number of questions of fluid-mechanical character. Authors concentrate attention on two alternative designs of droplets generation: one using centrifugal force and the other with liquid surface deformed electrostatically into a cone producing the droplets at its tip.

Keywords: additive manufacturing, droplets, molten metal.
\end{abstract}

\section{INTRODUCTION: ADDITIVE MANUFACTURING}

Hydrodynamics and thermomechanics of molten-metal drops in regimes of their generation by melting, flight ballistics, impact on solids, and solidifying are quite complex. Understanding them is necessary for producing metal objects by the additive method, already well developed and commercially available for handling soft and easily melted materials like polymers. Advantages of the additive approach are universality - there is no need of special tools - and fast-response adaptability. The latter means it is possible to change production simply by changing the computer program that controls the process. Particularly attractive are these advantages in producing spare parts. Manufacturing them long time before a arises a demand and storing them freezes in them sometimes considerable financial amounts - while all that has to be stored in the additive manufacturing approach is the computer program. Another potential advantage may be working with two (or even more) materials with mutually opposing mechanical properties (e.g. s hard and elastic), the continuous transition between them inside the product achieved by varying the percentage of the two deposited droplet kinds. Considering the general dependence of mechanical engineering on metals, there is a strong tendency to extend the additive manufacturing approach also to these materials [1]. The difficulties are mostly associated with the generally high melting temperatures and the consequent need of handling focused extremely powerful beams - laser or electron beam in vacuum. The latter is no serious obstacle since most metals need anyway some protection from atmospheric oxygen.

Due to the difficulties that must be overcome, attention so far concentrated on the two methods listed on the left-hand side of Figure 1, based on availability of the fine metal powders. Obviously, these methods require heating the metal twice to its melting temperature (first in producing the powder [2] and then in sintering) and this must be inevitably reflected in not really competitive economic aspects. Another problem, currently more important, is the limited mechanical strength of the sintered objects, substantially lower than that of compact material. Since metals are chosen as the material mainly for their capability to withstand high mechanical stresses, interest is currently turned towards the methods listed at the righthand side of Figure 1, handling molten-metal droplets instead of the powder particles. Proper accretion of droplets can make the manufactured objects practically equally strong as if they were made of fully compact material. 


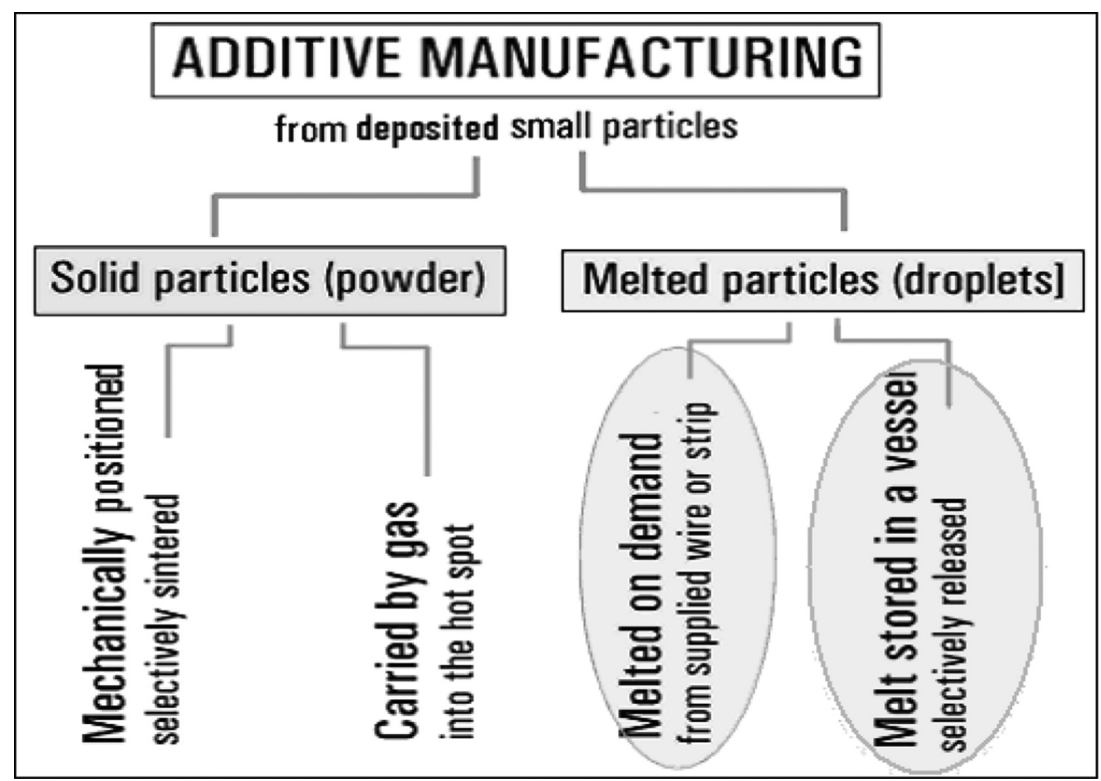

Figure 1: Principles of manufacturing metal objects by deposition of small particles. Of interest in this paper are the two methods working with drops and droplets (at righthand side).

Apart of the high temperatures and power levels, the next problem to be solved is the control of the drops ballistic flight. Gravity forces are too weak to play a really useful role. Proposed is action of electrostatic high-voltage fields.

Once the basic problems are mastered, the economy and productivity become the next questions of importance. Dependence on small gradually added particles to the manufactured product makes inevitably the process quite slow. The small size of the particles is dictated by considerations of product surface quality. Ideally, the additive manufacturing should avoid completely any subsequent steps of mechanical machining of locations requiring a fine surface. In principle the productivity may be increased by parallel operation of two or even more drop generators. This, however, would increase the investment price of the facility - and may lead to serious problems with their mutual mechanical motion interference.

\section{GENERATING MOLTEN METAL DROPS}

More promising way to productivity is increasing the repetition rate of drop production. The drops should be produced at such a rate that they follow one another in a quite dense chain, with separation between the chain members only a few drop diameters [2-4].

An interesting but so far not yet solved possibility would be switching between different drop size scales - working with quite large drops in the locations not demanding high surface quality. One of the problems of this approach may be the different laws governing the physics of larger drops and smaller droplets - Fig. 2.

Fortunately, compared with the standard atomisation as done nowadays with water or with liquid fuels, in the case of molten metals their distribution into smaller drops and droplets is generally easier. This is due to the typically low specific volume $\mathrm{v}\left[\mathrm{m}^{3} / \mathrm{kg}\right]$ (or its inverse, high density) of metals. Also the separation of the small molten amount of metal from the 


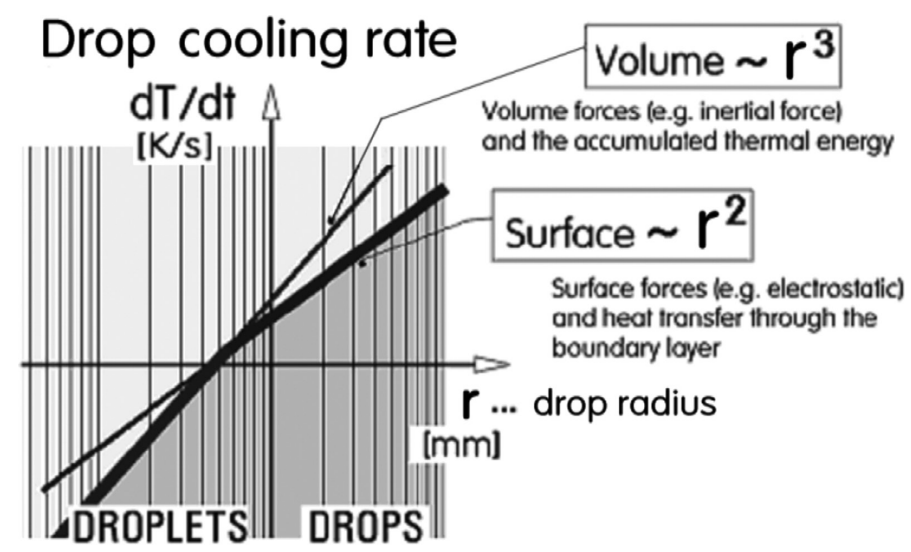

Figure 2: Drops differ from smaller droplets in the character of some of their governing laws. Typical example of the change is the drop heat removal rate in this diagram.
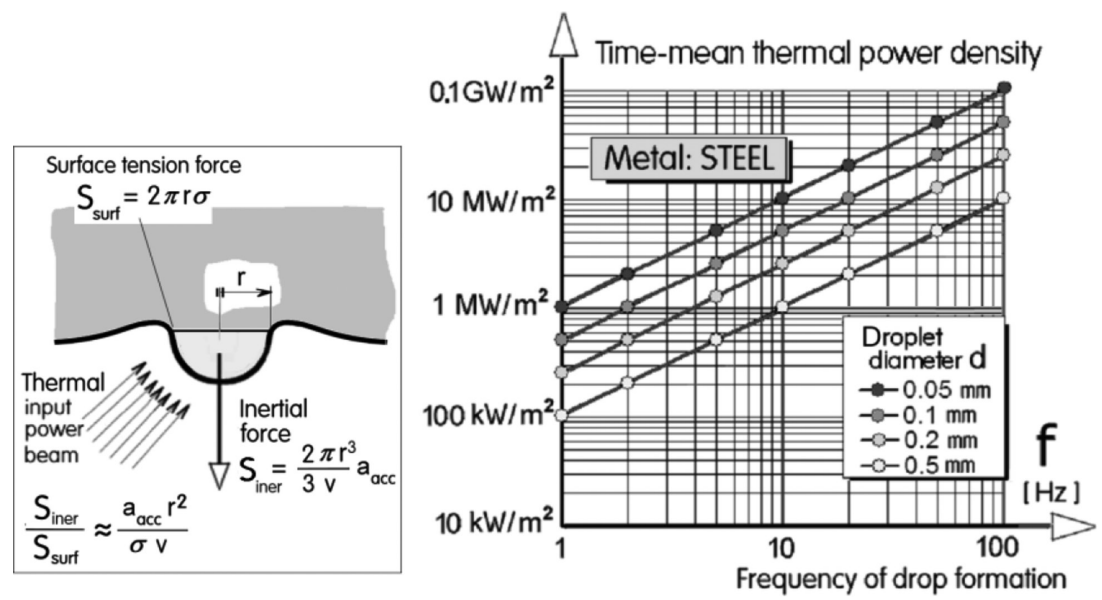

Figure 3: Basics of drop formation by melting metal surface. Diagram shows magnitude of thermal power required for repetitive drop production - ref. [5].

supplied solid material, schematically shown at left in Fig. 3, is rather easier. The key factor is the ratio of the separation-causing inertial force $S_{\text {iner }}$ (evaluated for the hemisphere drop in Figure 3) acting on the separated drop to the opposing surface force $\mathrm{S}_{\text {surf. }}$ As presented in this picture, of critical importance is the material property $\sigma \mathrm{v}\left[\mathrm{m}^{3} / \mathrm{s}^{2}\right]$ - product of surface tension $\sigma[\mathrm{N} / \mathrm{m}]$ and the specific volume. Values of this factor are presented in the diagram Fig. 4. Obviously, even for the lightest of metals the magnitude $\sigma \mathrm{v}$ is significantly lower than is the case of water.

Apart from influencing the drop separation in its generation, this lesser surface tension effect may cause rather unpleasant consequences, like the smaller tendency to achieve and maintain spherical shape. This may give rise to the phenomena presented in Figure 4. Both the formation of the dumbbell shapes as well as of the satellite droplets endangers achieving the desirable smoothness of the manufactured product walls. 

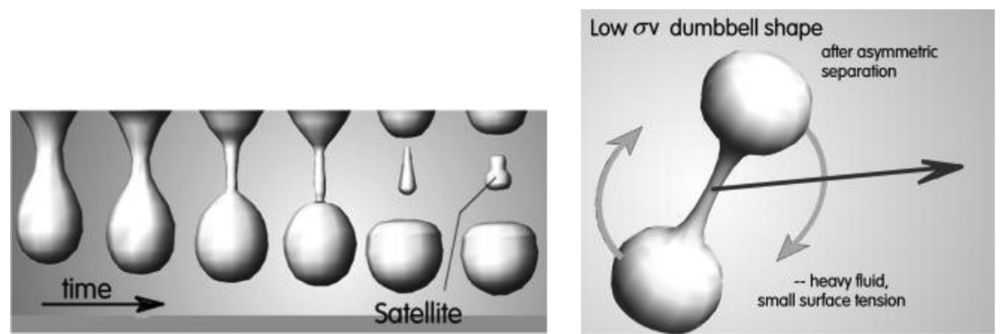

Figure 4: Product of $\sigma$ and $v$ is the critical material property (cf. left part of Figure 3) in several methods of metal drops (note the logarithmic scale).

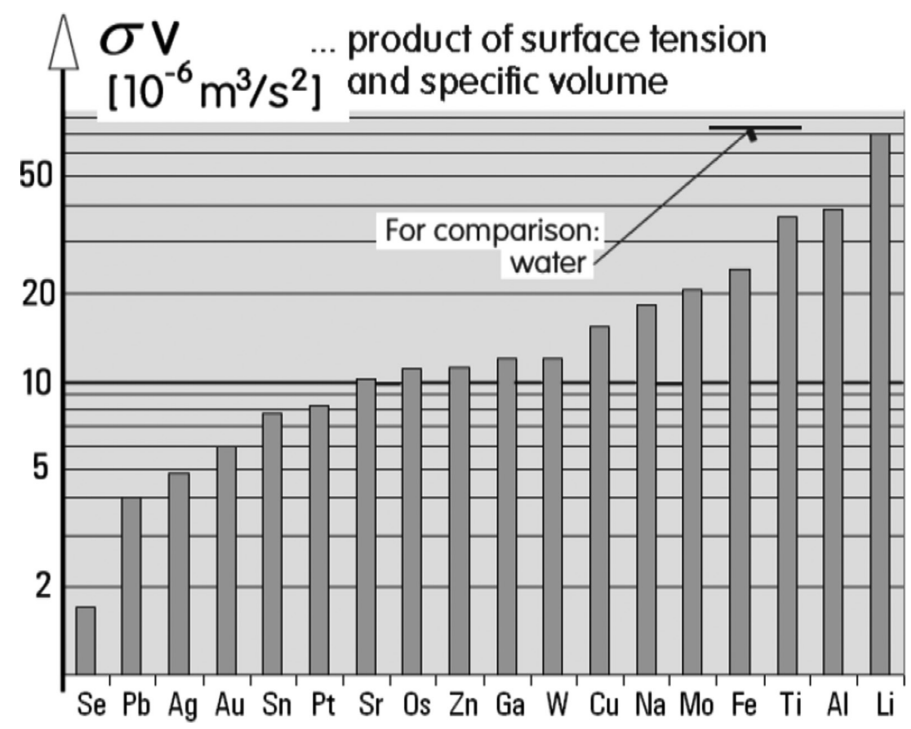

Figure 5: Problems encountered in drop generation are satellites and dumbbells moving differently than primary drops and disfiguring the resultant surface.

As shown in the time series at left in Figure 4, the disfigurations of the ideally spherical drop shape is caused by formation of the 'neck'. This contracts into a tread-like ligature, finally (fourth drop from left in Figure 4) breaking and leaving the satellite or smaller constituent of the dumbbell pair to follow its different ballistic trajectory. The difference of the trajectories is less important in the vacuum, and more pronounced if the oxidation is prevented by filling the internal space of the facility by an inert gas. Formation of satellite droplets depends on the magnitude of Ohnesorge number

$$
\mathrm{Oh}=\frac{v}{\sqrt{\sigma \mathrm{vd}}},
$$

where $v\left[\mathrm{~m}^{2} / \mathrm{s}\right]$ is molten metal viscosity (kinematic) and $\mathrm{d}[\mathrm{m}]$ is drop diameter. As a general rule, its is recommended to adjust the drop formation conditions so that the Oh values are within the limits $\mathrm{Oh}>0.1$ and $\mathrm{Oh}<1$. 
The diagram in Figure 3 provides an idea about the levels of time-mean power densities of the beams (beam of combustion product in the case of flames, of ions in the case of a plasma torch, of light in the case of a laser, and of electrons produced in an electron gun). Calculations assumed irradiating drops (or droplets) generated in a chain at the frequency f, assuming their separation distance equal to five diameters d. For simplification of the rather complex conditions it was assumed that regular absorption of incident power was on surface area of diameter equal to only one half of the overall drop diameter $d$ (remaining power being lost e.g. due to the reflection at a small incidence angle). At any rate, the power densities reaching the level of megawatt per $\mathrm{m}^{2}$ demonstrate the magnitude of difficulties to be overcome in molten metal droplet generation.

\section{MELTING ON CIRCUMFERENCE OF ROTATING DISK}

Metal material for producing the drops is usually supplied in the form of a wire the end of which is continuously moved to the focal point of the focused beam. Authors' new approach in their preliminary tests [6] have shown a distinct advantages of another configuration: melting the metal drop on the circumference of a thin disk of radius R, as shown in Figure 6, rotating at angular velocity $\omega[1 / \mathrm{s}]$. Once the drop separates from the disk, it is driven in the direction tangential to the disk surface by the force

$$
\mathrm{S}_{\text {iner }}=\frac{2 \pi r^{3}}{3 \mathrm{v}} \mathrm{R} \omega^{2}
$$

Reproducibility of the trajectories may be not sufficient and they are thus led in Figure 6 into a focusing system of electrodes, the first of them also securing additional electrostatic acceleration. Placing the gradually built manufactured product on the traversing gear, assumed in Figure 6, may be replaced by electrostatic deflection of trajectories by additional electrode plates.

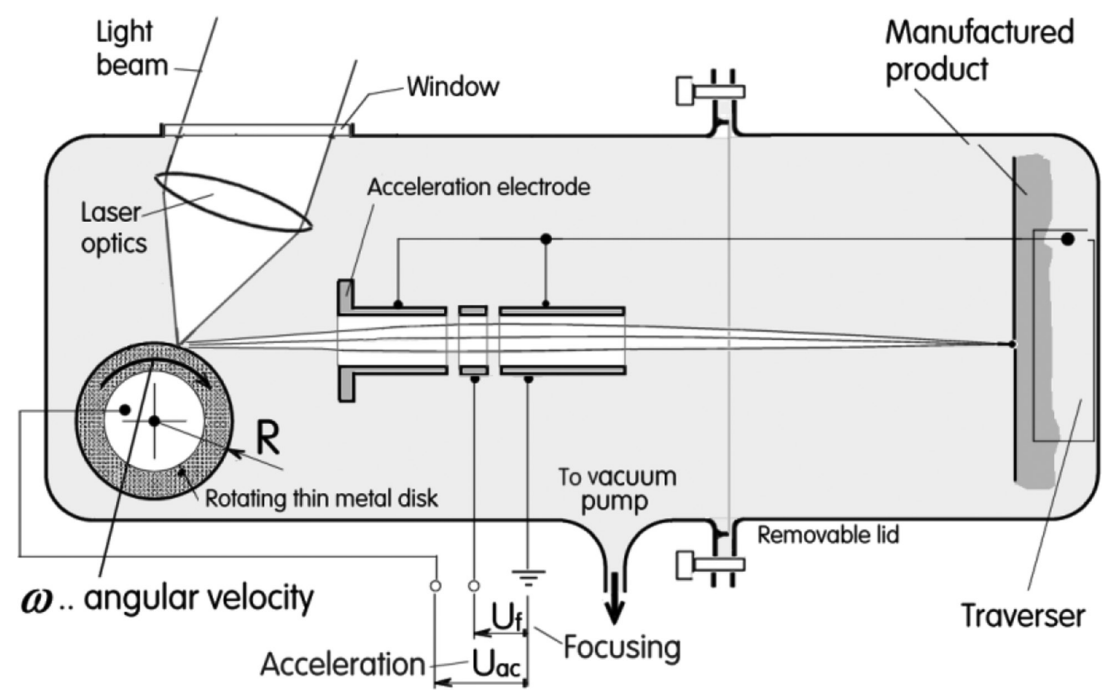

Figure 6: Principle of the principle of author's additive manufacturing test facility with centrifugal inertial force acting on formed drop - $[2,7]$. 


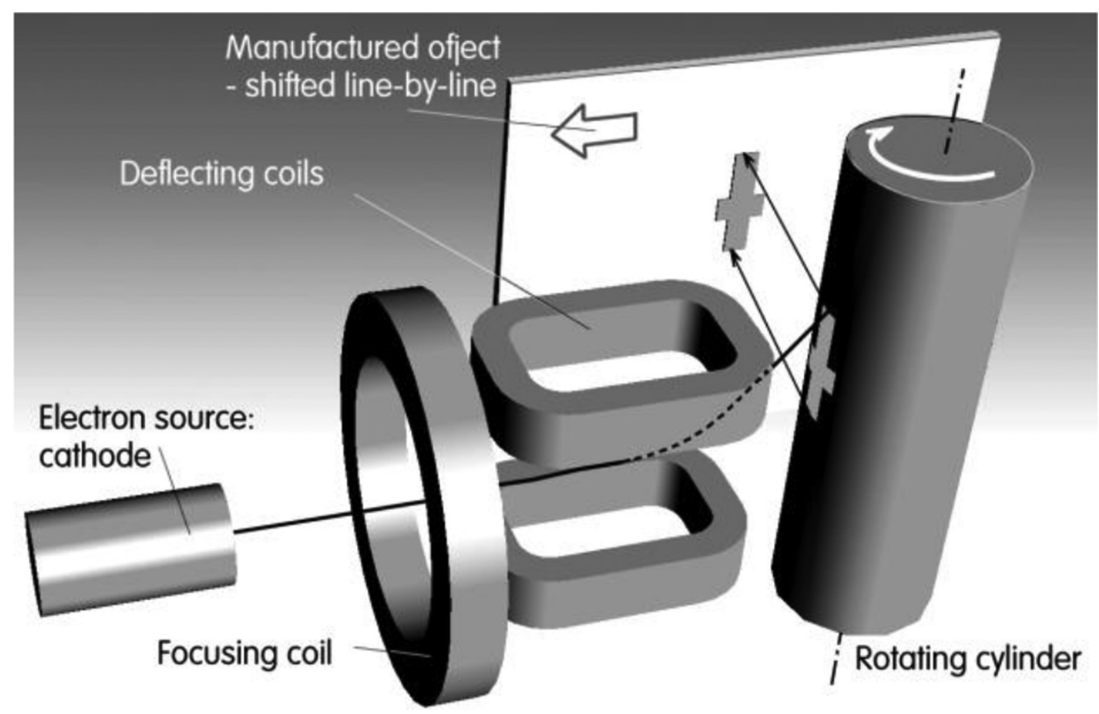

Figure 7: Principle of droplet formation on the surface of a wide cylinder by magnetically controlled electron gun $-[7]$.

In the next Figure 7 the principle of melting a rotating object circumference is extended to the melting focal points on the outer surface of a cylinder - the height of which is actually equal to the vertical extreme dimension of the product. This, of course, requires a high input power (some heating instead of the useful melting is lost by conduction inside the cylinder), provided by the electron beam. It is this beam, which is not only focused but here also deflected - electromagnetically, by the pair of coils.

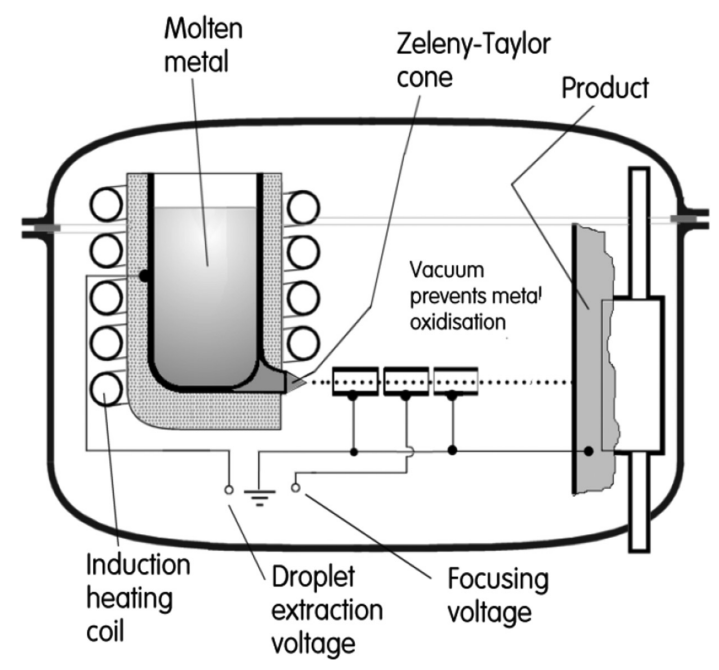

Figure 8: Schematic presentation of the additive manufacturing based on generating extremely small droplets at the tip of the Zeleny-Taylor cone [6]. 


\section{DROPLET GENERATION BY ZELENY-TAYLOR CONE}

In another proposal now under development by the present authors, shown in Figure 8, is addressed the problem of desirable two substantially different alternative drop size scales [3]. The large drops, intended for producing parts of the manufactured object not demanding a fine surface, are generated by a short flow pulse from a circular nozzle. If the product is moved by the traversing gear so that the instantaneous impact location should be empty, the nozzle exit is closed, as shown in Figure 9, by action of the surface tension. This aspect of the facility employs the generally large molten metal surface tension magnitudes, as listed in Figure 10.

The meniscus shown schematically as covering the nozzle exit in Figure 9 is deformed by the application of voltage $\mathrm{U}_{\text {acc }}$ into a conical shape. The phenomenon was discovered by Zeleny $[8,9]$, theoretically correct solution by the interplay of surface, viscous, and electrostatic forces was derived later by Taylor $[10,11]$, also $[12,13]$. If the voltage is increased, extremely small droplets are discharged from the cone tip. They may be guided and focused by the configuration of electrodes, Fig. 11 - using the shape of the field lines in the vicinity of the narrow gaps between the three components of the 'Einzellinse' electrode system.

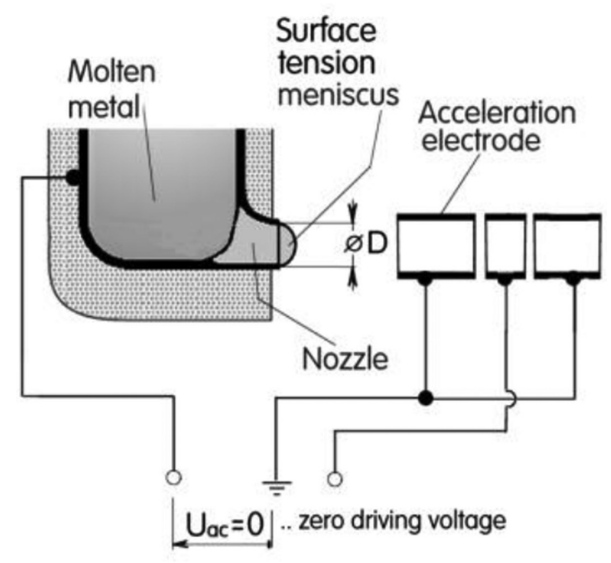

Figure 9: The nozzle exit in the OFF regime is blocked by surface tension.

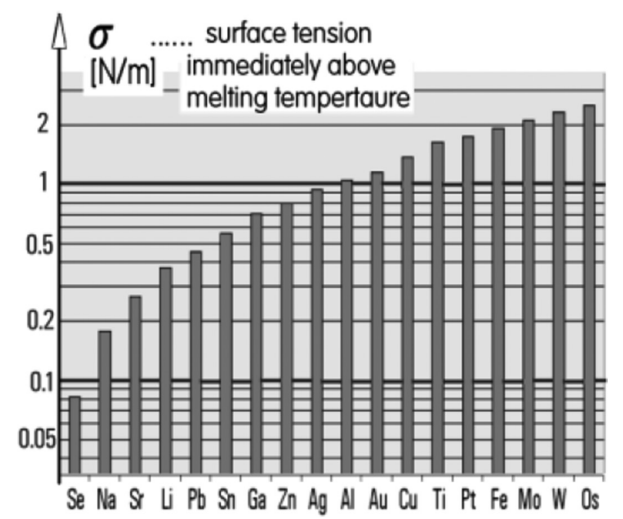

Figure 10: Surface tension values of molten metals vary in a wide range (note some very high values). 


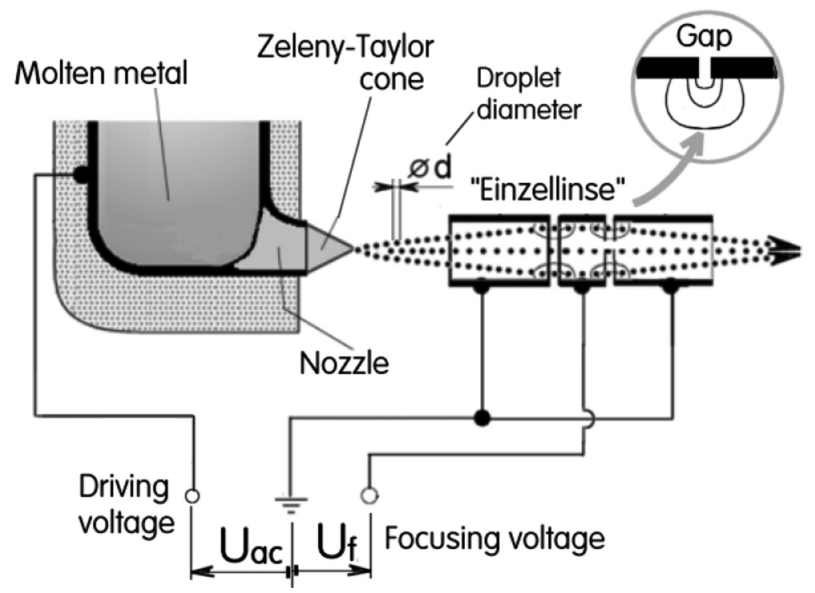

Figure 11: In the ON regime, extremely small (d $<<$ D, Fig. 9) droplets are discharged from the Zeleny-Taylor cone tip.

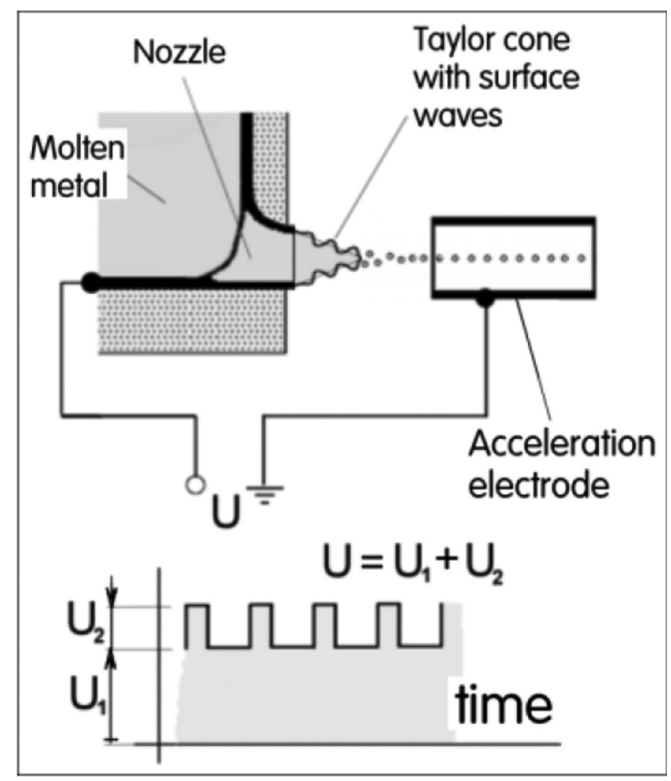

Figure 12: Schematically presented control of micro-droplets generation by applied periodic voltage component $\mathrm{U}_{2}$ - producing waves on the cone surface.

Interesting and useful effect was recently found, as shown schematically in Figure 12: if the driving high voltage contains periodic peak component $\mathrm{U}_{2}$, on the Zeleny-Taylor cone surface are formed waves travelling towards the cone tip with increasing amplitudes. In the end, when the waves move to the tip, they are separated from it in an orderly manner. Highspeed camera images of the cone tips (at left) as well as the ring electrode rim (at right-hand side) in Figure 13 show three different phases of the molten metal drop formation. 


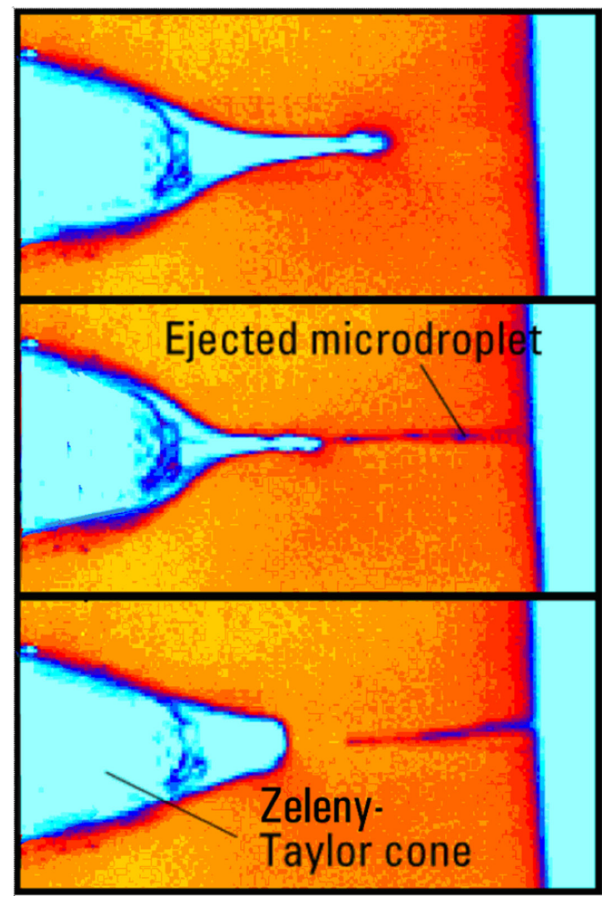

Figure 13: Three high-speed camera images of metal microdroplets ejection from the cone tip.

\section{CONCLUSIONS}

Additive manufacturing - as opposed to the traditional subtractive one, with material removal from the manufactured object - promises to bring a new dimension even to producing metal objects, especially those of complex shapes. Until recently, the progress with the use of metal as the material has been slowed by the attention given to the less demanding sintering approach. Nevertheless, the pressure of the demand for solid-metal objects and gradual mastering of the problems associated with generation and handling of the molten-metal droplets indicate feasibility of success being within reach. Authors present two alternative configurations used in their current tests.

\section{ACKNOWLEDGEMENTS}

First author gratefully acknowledges support by grant 13-23046S received from GAČR - the Czech Science Foundation. Both authors also received institutional support RVO: 61388998

\section{REFERENCES}

[1] Alexander W. \& Street A., Metals in the service of man, Penguin paperbacks, 1999

[2] Dunkley, J.J. \& Aderhold, D., Centrifugal atomisation for powder production. Proceeding of Euro PM 2005, 2, Prague, p, 9, 2005.

[3] Tesař, V., Fluidic control of molten metal flow. Acta Polytechnica-Journal of Advanced Engineering, 43, p. 15, 2003.

[4] Vader S., Vader, Z., Karampelas, I.H. \& Furiani, E,P., Magnetohydrodynamic liquid metal jet printing. TechConnect Conference, Washington D.C., June 2015. 
[5] Tesař, V., Fluid mechanics of additive manufacturing of metal objects by accretion of droplets - a survey. European Physical Journal Web of Conferences, 114, paper Nr. 02122, 2016.

[6] Šonský, J. \& Tesař, V., Method and facility for manufacturing metal products. Czech Patent Application, PV 2016-227, filed Apr. 2016.

[7] Šonský, J. \& Tesař, V., Method and facility for producing metal products by gradual addition of small particles having character of molten drops, Czech Patent Application, PV 2016-328, filed Jun. 2016.

[8] Zeleny, J., On the conditions of instability of liquid drops, with applications to the electrical discharge from liquid points. Proceeding of Cambridge Philosophical Social, 18, p. 71, 1915.

[9] Zeleny, J., The electrical discharge from liquid points, and a hydrostatic method of measuring the electric intensity at their surfaces. Physical Review, 3, p. 69, 1914.

[10] Taylor, G.I., Disintegration of water droplets in an electric field. Proceeding of the Royal Society A, 280, p. 383, 1964.

[11] Taylor, G.I., Electrically driven jets. Proceeding of the Royal Society of London A: Mathematical, Physical \& Engineering Sciences, 313, p. 453, 1969.

[12] Fernández de la Mora, J., The fluid dynamics of Taylor cones. Annular Review of Fluid Mechanics, p. 217, 2007.

[13] Wilm, M.S. \& Mann, M., Electrospray and Taylor-cone theory, Dole's beam of macromolecules at last?. International Journal of Mass Spectrometry and Ion Processes, 136 p. 167, 1994. 